\title{
La participación de América Latina en el sistema económico internacional de la postguerra*
}

\section{INTRODUCCIÓN}

Este trabajo constituye la primera parte de una serie de estudios destinados a analizar determinados aspectos de las relaciones económicas y politicas internacionales de algunos paises de América I atina, cn un perlodo histórico específico.

La inquietud sobre esta materia se fundamenta en el deseo de profundizar, desde el ángulo latinoamericano, respecto a dos conjuntos de elementos de relaciones internacionales, que han incidido y condicionado, en forma importante, la conducta internacional de los países de América Latina y la búsqucla de posiciones de una cierta autonomía e independencia.

El primer conjunto de elementos está constituido por la estructura. ción de un orden económico internacional, al término de la Segunda Guerra Mundial, tema que serí abordado en este ensayo introductorio, y en un segundo trabajo en que se analizarán las organizaciones económicas de nivel mundial (restringidas en la práctica al

*El presente trabajo es parte de una investigación mís amplia que el autor desarrolla con el apoyo del Social Science Research Council (F.uu.), institución a la que se agratece la colaboración.

Esta insestigación ha sido inscrita all los programas del Centro Intcruniversitario de Desarrollo Andino (CiNoA) y constituye uno de los trabajos con que este organismo participa en el RIAs. (Programa de Relaciones Interuacionales de América Latina).

El autor desca agradecer al I'rofesor de la Universidad Católica de Valparaíso, sr. Manfred Wilhelmy, su coopcración en la preparación de este trabajo, ya sea mediante sus valiosas sugerencias y críticas como asimismo, su contribución en el señalamiento de una parte: de la bibliografia. 
sistema occidental, con la excepción de UNCTAD y las tendencias recien. tes en cl Banco Mundial y otras organizaciones).

El segundo conjunto de elementos está constituido por las relaciones horizontales entre los paises de América I atina. Si se considera que hasta 1960 (fecha del Tratado de Montevideo) las relaciones conómicas cle estos paŕses se circunscribím al ámbito bilateral, parce necesario profundizat las politicas de comercio exterior $c$ inversiones de algunos patíses. A este tema y al estudio de la organización internacional conómica en la región nos proponemos dedicar dos ensayos adicionales.

Conviene ya, en este momento, situar el aspecto que nos mueve fundamentalmente a reflexionat sobre el tema. Parece necesario decir. al mismo tiempo, que estas reflexiones se insertan en realidades contingentes y actuales. En una palabra, queremos expresitr que, si bien algumos aspectos histórico-institucionales retienen muestra atención, no son los principales.

I a esencia de nuestra inquictud radica en la implantación y consolidación de una lilosofia económica internacional, destinada a regular de una manera homogénea el conjunto de los aspectos más determinantes de las políticas exteriores de los países que participaron on el sistema.

La adhesión a, y el seguimiento de cstas políticas miversales no traía consigo. obviamente, una neutralidad de acción y posición. Por cl contrario, las resultantes lógicas de determinadas acciones debían reflejarse paulatinamente en el establecimiento de nucvas políticas de cooperación y relación, con especial inciclencia en ventajas derivadas de acercamientos geográficos y complementaciones económicas naturales.

Analizando la situación desde un ángulo latinoamericano, parece neccsario observar que, salvo en mínima parte, la ałhesión a los principios universalmente aceptados ha sido de carácter formal. I.o curioso del fenómeno es que esta adhesión formal (materia que se desarrollatí en otro trabajo, mostrando los contrastes entre lo decidido y las políticas adoptadas en materia de comercio internacional e inversiones) comicna a tramsformarse, en el momento actual, en aplicación concreta de principios de acción en un número importante de páses latinoancricanos, sin que esto sea el efecto de determinadas posiciones comunes. Al contrario, son las realidades típicamente nacionales las que han condicionado efectivamente la vuclta a la adopcion de soluciones imaginadas también luego de la Segunda Guerra Mun- 
dial para los páses latinoamericatros pero que, como se dijo, no recibieron explicación príctica iniciat ${ }^{1}$.

Lucgo de la Segunda Guerra Mundial (y aun antes de su terminación) en el seno de los aliados comenzatron a diseñarse instrumentos e instituciones universales tendientes a asegurar la par. Junto a las conocidas instancias políticas que constituyen la hoy llamada familia de las Naciones Unidas, se intentó establecér instituciones reguladoras de la problemáticat economica internacionte por considerase que, parte importinte de las ausas que llevaron a los dos conflictos mumdiales vividos en los primeros 45 anos del siglo $\mathrm{xx}$, fucron de carácter económico.

Esto implica la imposición de un criterio, de evolnción lenta, en el sentido de entregier a organismos internacionales la regulación y control de determinados aspectos de las políticas económicas macionales.

Es necesatrio advertir que esta temdencia de internacionalización en la conducción de las políticas coonomicas nacionales tiene un origen perfectamente detectable, en forma concreta, en la Primera Gucrra Mundial.

En efecto, es notitble conocer la evolución de instituciones comu. nes luego de la Guerra del 14 al 18 y su prolongacion posterior al periodo de postguerra de 1939 y su implementación efectiva luego del estallido del conflicto:.

Ia filosofía conómica que se abre camino luego de la Segunda Guerra, se basa en determinatats premisas que fueron adoptadas como intrínsecamente válidas y que, por otrat parte, constituyeron un elemento de reacción a la situación de preguerra: se trataba precisatmente de hacer todo lo contrario a lo vigente Instat el inicio de las hostilidades.

Ia investigación se dirige al estudio del funcionamiento real de determinados acuerdos, tanto de akance globit, al menos en prin.

'parece no caber duda que las polfticas de apertura a los mercados extemos practicadas por Brasil y mis recientemente por Argentina. Urbaray y muy principalmente, Chile, coinciden, en lo que a este ingules se refiete, con los postulados de climinación universal e indiscrimiladia (razón del origen) de los obstáculos al comercio intentacional y a ha libre circulación de capitales.

En otras materias, como es el caso de la cooperación para superar los problemas del desempleo nacional, la idemtidacl mo parece tat.

"Una narración completa y detallada puede verse en Jean Monnet, Memoires, paris, Fajard, 1976, especiahnesuce los capítulos 3 (La acción común) y el capítulo 7 (El programa para la V'ictoria) de la Primera Parte. 
cipio, como propios a América Latina, suscritos por los distintos países. Algunos de ellos llegaron a ser instrumentos jurídicos con fuerza obligatoria; otros, en cambio, no fueron adoptados por los estados.

Entre los últimos se destaca la Carta de La Habana, que disponía el establecimiento de la Organización Internacional de Comercio. Derivada de esta última y en íntima implicación y operativa, debe mencionarse el gatT o Acuerdo sobre Tarifas y Comercio.

Se atribuye en esta investigación una decisiva importancia a la revisión de los postulados y contenidos de la Carta de I a Habana. En efecto, consideramos que de este documento pueden extraerse válidamente los elementos que caracterizaron una filosofía económica internacional que, de una $u$ otra manera, de una forma más o menos perfecta, han dirigido la acción de gobiernos y empresas hasta un pasado relativamente reciente.

Al mismo tiempo pueden extraerse de los principales documentos, antecedentes que permiten tener una visión relativa de las posiciones que adoptaron los países latinoamericanos participantes ${ }^{3}$. Es de señalar en forma muy principal que, analizada esta materia tomando en consideración la participación en los debates de dos tipos de países, es decir, los industrializados y los que no lo son, o si se quiere mejor, los desarrollados y los menos desarrollados en aquella época, debe observarse que fueron principalmente los países de América Latina los que hoy podrian ser denominados como representantes del Tercer Mundo*. Ello implica aceptar que sus actuaciones debieron producir resultados en la acción internacional posterior.

Este estudio no analizará las distintas etapas que se siguieron para llegar a determinados acuerdos. Ello ha sido objeto de una interesante literatura y presenta, principalmente, un carácter histórico.

"Ver nRown (W.A.), The United Slates and the restoration of World Trade, The Brookings Institution, Washington D.C., 1950.

La Documentación française, La Charle de la Havane (analyse et commen. taires), Paris, 1948.

'Los países que participaron en el establecimiento de la Carta de La Habana fueron: Afghanistan, Argentina, Australia, Austria, Bélgica, Birmania, Bolivia, Brasil, Canadá, Ceylan, Chile, China, Colombia, Costa Rica, Cuba, Dinamarca, República Dominicana, Egipto, Ectuador, Estados Unidos de América, Francia, Gran Bretaña e Irlanda del Nortc, Grecia, Guatcmala, Haití, India, Indonesia, Irak, Irán, Irlanda, Italia, Libano, Liberia, Luxemburgo, México, Nicaragua, Noruega, Nueva Zelandia, Pakistáu, Panamá, Países Bajos, Perú, Filipinas, Po. lonia, Portugal, Rhodesia del Sur, Salvador, Suecia, Suiza, Siria, Checoslovaquia, Transjordania, Turquáa, Unión del Africa del Sur, Uruguay y Venezuela. 
Ia institucionalidad económica internacional está fuertemente condicionada por factores de orden político. El objetivo de esta sección es, primero, identificar y describir los procesos polf́ticos internacionales que, desde antes de la Segunda Guerra Mundial, cristalizaron en un arreglo institucional básico, fundamentalmente limitado a las potencias occidentales, bajo la conducción de Estados Unidos como potencia dominante.

En una segunda sección del estudio se examinará la inserción de América Latina dentro del sistema internacional de postguerra, enfocando especialmente las condicionantes de orden político-social y externo de la participación internacional de la región, con énfasis en el período de 1945 a 1960 , aproximadamente.

Ambas tareas son indispensables para situar correctamente el papel de los estados latinoamericanos en la gestación y funcionamiento del sistema económico-político internacional de los últimos treinta años, y para el posterior análisis de papeles nacionales en las organizaciones internacionales económicas.

A. El fin de la Segunda Guerra Mundial y el nuevo orden emergente.

El sistema internacional hacia el fin de la Segunda Guerra Mundial evolucionó hacia la formación de dos polos de poder mundial. Europa Occidental, después de más de cuatro siglos de dominación del sistema internacional, quedó relegada a una condición de fuerte dependencia frente a Estados Unidos para su defensa y reconstrucción económica. Desde la segunda mitad de la década de 1950, Europa recuperó en parte su posición económica, en gran medida debido a los progresos hacia la formación de un mercado común y a la extraordinaria "performance" de la economia de Alemania Federal. En la década siguiente, comenzó a darse una mayor participación europea en el plano estratégico-militar, fundamentalmente asociada con las crisis provocadas por la política exterior y militar del régimen degaullista en Francia, y con el surgimiento de Alemania Federal como el principal contribuyente al esfuerzo militar convencional de la otan.

A pesar de este resurgimiento parcial, puede afirmarse que la estructura bipolar se mantuvo básicamente vigente, por lo menos hasta bicn avanzado el hoy debatido proceso de distensión. La razón de esta permanencia relativa debe buscarse directamente en los proyectos políticos planteados al interior de la alianza británico-norte- 
americana-sovićtica desde antes del final de la guerra, y en el triunfo de hecho de la posición de las "esferas de influencia" como principio ordenador de las relaciones de poder frente a la posición "universalista". La primera era sustcntada por la Unión Soviética, contando en cicrto modo con la simpatía de Gran Bretaña, mientras la segunda constituyó la tesis oficial de Estados Unidos.

En la perspectiva de la posición universalista, el logro de una paz estable en cl sistema de postguerra debia depender del ejercicio de responsabilidades compartidas por las grandes potencias en la con. ducción del sistema internacional y en los subsistemas regionales. La superación de los mecanismos de bloques y alianzas característicos de las décadas anteriores resultaba necesaria para el mantenimiento del orden que se lograra una vez terminadas las hostilidades. Este resurgimiento, a su vez, debía obligar a definir fórmulas de gobicrno, especialmente en los países vencidos y en los que se encontraban en proceso de liberación, debiendo los respectivos arreglos políticos contar con el acucrdo de las grandes potencias.

Para la posición favorable al establecimiento de las "esferas de influencia", el objetivo de la cstabilidad internacional no podía ser logrado sino asignando responsabilidades claras y definidas a cada una de las grandes potencias en áreas geográficas determinadas. Si bien no se negaba a nivel globitl y en forma teórica la responsabilidad comparticlat y conjunta de las grandes potencias encargadas de la estructuración del sistema de postgucrai por mantener la paz del mismo, los partidarios de esta posición veían solamente uma fuente de conflictos internacionales interminables en el requerimiento de conciliar intereses - que probablemente serían contrapuestos- en cada estado no dominante. Por consiguiente, las fórmulas internas de gobierno, la composición de los mismos, y especialmente sus políticas exteriores, debian definirse de manera congruente con las realidades del pocler en cada región, y por tanto siguiendo los lineamientos de las respectivas potencias dominantes. salvo que por acuerdo de éstas pudiera intervenir más de una gran potencia en los asuntos de determinado estado, caso excepcional en el cual deberían realizarse consultas entre las grancles potencias interesadas $5^{5}$.

Estos principios opuestos habrian de tencr importante gravitación

"Ia obra de Ludwig Dchio. The Precarius Balance, Four Centuries of the European Pourer Struggle, Nueva York, Vintage Books, 1962, describe la historia del sistema internacional en términos de succsivos "retos" a la estructura interestatal vigente en cada época. Pata Dehios, la clinimica fundameutal del sistema 
en la estructuracion de las organizaciones internacionales mundiales y regionales. En efecto, al mantenerse la vigencia formal del principio universalista en la conformación del sistema de las Naciones Unidas, se reconoció la necesidad de alcanzar un consenso básico de todas las grandes potencias como condición sine qua non del funcionamiento satisfactorio de los mecanismos fundamentales de la organización internacional, especialmente por su puesto el Consejo de Seguridad ${ }^{\text {. }}$ Al mismo tiempo, sin embargo, la cstructuración del sistcma en bloques hizo que lat premisa universalista quedase de heclio negada, limitándose así las posibilidades de funcionamiento del sistema de Naciones Unidas. En cl Consejo de Seguridad, la política de bloques se manifestó a tlavés del veto, mientras que en las organizaciones cconómicas - Banco Internacional de Reconstrucción y Fomento, Fondo Monctario Internacional y Acuerdo General de Aranceles y Comercio, fundamentalmente - la política de bloques se dio a través de la no participación de los países socialistas ${ }^{7}$. En vez de constituir

se da en la gradual ampliación del ímbito internacional y en la reafimación de las estructuras descentralizadas luego dol fracaso de cada pretensión hegemónica. In tereer elemento de ordét pinpolítiso consiste en la permanente tensión cutre potencias "costlinmtiale" y "maritinas". Dentro de esta concep-

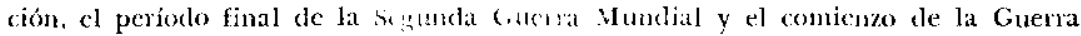

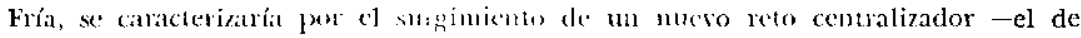
la Unión Soviética-- a las poteincias recidentites. Fn este enfremamituto, "Europa ya no es el centro y su sistema yit no es la estuctura principal de la política mundial". (Dehio, op. cit, p. 272). Pero se mautiene la oposición geopolítica, a la que se agrega una dimension ielesológica, ya que "uma vez más, el espéritu extraño de la politica de poder continental estabia obstaculizando el paso a un orden democratico mundiat. Pero est:t ve iste venta, no del territorio de la vicja Furopa, sino, de mancta más amenarante, de las profundidades de Eurasia". (Dehio, op. rit., p. 273).

En el libro de Geofficy Batraclough, An Introduction to Conlemporany His. tory, Londres, C. A. Walts, 1964, se ofrece uIl completo patıorama del surgimicuto de las potencias y movimientos politicos no occidentales en el curso del prescnte siglo.

"En la historia diplomitica de Herbert Fcis, Churchill-Roosevelt-Stalin, The War they Waged and the Peace They Songht, Princeton, N. J. Princeton University press, 1957, se trata extensamente el desamollo de las dos posiciones en el seno de la alianza occidental y la trss. Para una síntesis, véase Louis J. Halle, The Cold War as History, Nueva York, Harper \& Row, 1967.

Sobre este pumto, ve' Inis Clatule, Swords Into Plowshares, Nueva York, Ramdom House, 1964.

'Fr el caso del gatr se fuc produciendo la incorporación paulatina de algunos paises socialistas europeos. El caso yugoslavo constituye una situación diferente que no podemos analizar aquí. 
genuinas organizaciones de alcance universal, las principales organizaciones económicas internacionales del sistema de Naciones Unidas pasaron a constituir expresiones institucionales de la organización internacional del bloque occidental, conducido por Estados Unidos.

El "vacío de poder" creado por el colapso de Europa fue rápidamente llenado por la Unión Soviética y Estados Unidos, potencias dominantes principales del sistema de postguerra. las motivaciones, objetivos y estrategias de ambos estados han sido extensamente discutidos en la literatura especializada, atribuyéndose a uno y otro sistema designios imperialistas o conservadores, orientaciones pacíficas o belicistas, etc., dependiendo tales juicios en medida significativa de las preferencias ideológicas de los observadores. Respecto de Estados Unidos, el nuevo centro de gravedad del sistema internacional después de un largo período de participación relativamente marginal en el campo internacional, se ha afirmado por algunos que buscó establecerse como potencia rectora del nuevo orden, mientras otros ven en el nuevo papel norteamericano una consecuencia inevitable, mas no un objetivo conscientemente perseguido a través de su participación en los eventos de la década de 1940. Por otra parte, mientras para unos la uRss se encontraba resucltamente encaminada a la formación de un imperio de alcance mundial, para otros el imperialismo soviético obedecía a motivaciones en parte defensivas, que ya se encontraban en la tradición de la política exterior zarista, entre las que destaca el deseo de proteger el territorio nacional frente a invasiones desde Occidente, potencialmente facilitadas por la carencia de fronteras naturales del territorio ruso ${ }^{8}$.

No es posible abordar en el presente contexto el complejo problema insinuado, que entronca en el debate sobre el origen de la Guerra Fría, período siguiente a la destrucción de la alianza bélica victoriosa, más allá de señalar la existencia de estas perspectivas analíticas alternativas sobre el comportamiento de los contendientes, que necesariamente deben llevar a conclusiones opuestas. Es necesario, sin embargo, presentar un punto de vista general acerca de la reorientación de la política internacional al comienzo del período en estudio, puesto que la situación internacional de Anérica Latina se vio decisivamente afectada por ésta, dependiendo las conclusiones especiflcas a que se llegue acerca de la región de la visión global que se adopte en este problema.

En general, el punto de vista según el cual Estados Unidos se vio

${ }^{8}$ Esta motivación es destacada entre otros, por Halle, op. cit. 
prácticamente impelido a asumir un papel internacional de conducción, sin que hubiera mediado una reorientación consciente de la política exterior, parece conducir a una interpretación equivocada o por lo menos distorsionada del papel internacional norteamericano y de las relaciones con Amćrica Latina en Ias décadas siguientes. Si bien puede sostenerse que la entrada de Estados Unidos en la Segunda Guerra Mundial resultó de circunstancias imprevistas para los gobernantes norteamericanos, no ocurrió lo mismo con la participación en el sistema de postguerra, que fue largamente debatida y, una vez producido un consenso básico en Washington, planificada, negociada e implementada al interior del gobierno, de la alianza de guerra, del nuevo sistema de Naciones Unidas y el sistema interamericano. El grupo que logró imponer su critcrio y tomó la conducción de la política de postguerra fue el llamado sector "internacionalista" de la élite norteamericana, grupo que rehusó en todo momento volver al pasado aislacionista y sostuvo que solamente una participación rectora de Estados Unidos en el nuevo sistema internacional era compatible con los intereses nacionales básicos. Los "internacionalistas" vcían en realidad un imperativo de participación global de Estados Unidos, aunque su lenguaje y las justificaciones de las nuevas políticas fueran frecuentemente defensivas y "reactivas". Conductores e inspiradores de la diplomacia norteamcricana del período en cstudio tales como Dean Acheson, George Marshall y George Kennan, consideraban que solamente una "visión realista" del sistema internacional podía conducir a la formulación de políticas racionales al servicio de los intereses norteamericanos, definidos en función de relaciones de poder más que de consideraciones de orden ideológico. La "doctrina de la contención" elaborada por Kennan enfatizaba la necesidad de detener el avance geopolítico soviético, recurriendo a medios políticos siempre que fuera posible, pero sin excluir la aplicación de la fuerza en puntos geográficos determinados cuando así lo exigieran las circunstancias. Esta fórmula de Kennan representó un cambio de criterio respecto a la formulación -también en 1947- de la llama. da "doctrina Truman", según la cual el interés fundamental de Estados Unidos era la defensa del sistema liberal democrático de Occidente contra los peligros dictatoriales y totalitarios, lo que en la práctica se entendió referido solamente a los regímenes comunistas. Obviamente, la opción entre el "realismo" de Kennan, centrado en las relaciones de poder y en la necesidad de alcanzar un equilibrio global basado en el papel preponderante de Estados Unidos, y el 
"idealismo" defensor de las democracias occidentales, era sumamente importante para la orientación global de la diplomacia así como para la conducción de políticas específicas. Si bien nunca hubo un pronunciamiento al respecto -y no podía haberlo- en la práctica prevaleció el criterio de Kennan, implementándose una política de contención definida en función de relaciones de poder más que de preferencias ideológicas. Esto hizo posible, por ejemplo, la adopción de criterios flexibles para enfrentar situaciones especiales que no calb́an dentro de la lógica de la doctrina Truman, pero en que había en juego consideraciones estratégicas importantes, por ejcmplo, en cl caso yngoslavo ${ }^{9}$. Tal política de contención tuvo su expresión en los sistemas de alianzas organizados alrededor del mundo a partir de 1947, y en los pactos bilaterales que los complementaron. Uno de sus sul)sistemas del campo occidental era, precisamente, el sistema interanericano, fundado en sus aspectos de seguridad en cl Tratado Interamericano de Asistencia Recíproca o Tratado de Río, de 1917, y en los pactos militares bilaterales entre los miembros latinoamericanos del sistema y Estados Unidos ${ }^{10}$.

B. Criterios orientadores y caracteristicas del orden intemarional de postguerra.

En primer lugar, para situar correctamente esta materia, convicne referirse a la acción de determinados pátíses que, en forma vital, transmitieron determinados conceptos y posiciones político-económicas al conjunto de las naciones que participaron en el estitblecimiento de este orden.

En especial, la organización del orden cconómico intcrnacional de postguerra debía seguir los lineamientos del internacionalismo propugnado por los grupos rectores de la diplomacia norteamericana. Debe recordarse que al periodo de libertad económico internacional del siglo $\mathrm{xIx}$ y comienzos del presente siglo siguió, en el período hasta el fin de la Segunda Guerra Mundial, una estructura de bloques económicos más o menos rígidos, caracterizados por su orientición

"Véase al respecto Halle, op. cit., y las memorias de Dean Acheson, Present at the Creation, Nueva York, New American Library, 1969.

${ }^{30}$ Algunos han sotenido, sin embargo, que la política te alianzas habria sido llevada al extremo de constituir una verdadera "pactomanía" cn la década de 1950 mientras John Foster Dulles fue secretario de Estado. Tal "manía", se sos. tiene, habría sido una distorsión de los objetivos originales de la política de contención de Kennan. 
proteccionista y su organización alrededor de potencias dominantes como Inglaterra en el Imperio Británico ${ }^{11}$ y Alemania en el sistema económico de guerra, edificado sobre la base de las conquistas bélicas en Europa.

Para el Secretario de Estado del Presidente Franklin D. Roosevelt, Cordell Hull, el restablecimiento de un sistema económico internacional basado en el libre comercio era indispensable para garantizar la paz y prosperidad internacionales, asi como los intereses fundamentales de Estados Unidos en la economía internacional.

Esencialmente, esta posición implicaba la posibilidad de participar de manera activa en el proceso de inversión internacional.

Lo anterior debe comprenderse a la luz del desarrollo del sistema cconómico norteamericano, en cuyos círculos rectores había considerable interés en facilitar la acción de la empresa privada norteamericana en el ámbito internacional. Esto requirió, en ese momento, reemplazar el caos prevaleciente por un sistema multilateral estable y que mediante bases no discriminatorias, le permitiera competir en términos equitativos.

En primer lugar, apareció necesario buscar las formas para el incremento del volumen y la calidad del comercio internacional.

La implantación de los nuevos principios no fue tarea fácil. Algu. nos países con una importante contribución en el comercio internacional de la época, si bien comprendían teóricamente las ventajas de un sistema multilateral abierto, se resistían a abandonar por completo determinadas técnicas de control y la utilización de los mecanismos de relación bilateral vigentes en ese momento.

Esto hizo que se manifestaran, desde el inicio, la dialéctica de establecer por una parte, un mccanismo multilateral y dejar vigente, al menos en parte, la capacidad de acción negociadora bilateral,

${ }^{11} \mathrm{La}$ organización del bloque o área de la libra esterlina fue una de las principales medidas tomadas por Gran Bretaña para enfrentar la depresión de comienzos de la década de 1930. En csta área participaron los miembros del Imperio Británico, con la excepción de Canadá, además de algunos otros páses fuertemente vinculados a Gran Bretaña por lazos comerciales y financieros. El objetivo inglés al organizar este bloque fue proteger el comercio imperial en el clima económico intcrnacional altamente competitivo de la época. El área de la Iibra funcionó sobre la base de la mantención de reservas internacionales en libras, el uso de la libra como medio de pago internacional en el área, y la mantención de paridades monetarias en relación con la libra. Después de la Segunda Guerra Mundial, el uso de la libra fue retrocediendo, pero debido a la existencia de reservas en esa moneda en diversos países, el área debió mantenerse en existencia. 
especialmente arraigada en las concepciones y las prácticas políticoeconómicas europeas. Especial temor se tuvo a tener que soportar, de una u otra manera, eventuales inestabilidades ajenas.

Precisamente es en este punto que el sistema que se estableció innovó. Si bien no se podía eliminar la exposición a las inestabilidades extranjeras, la prescripción adoptada establecía la necesidad de crear los mecanismos cooperativos para paliarlas. De allí la necesidad de reglas objetivas y de aplicación universal para hacer frente a las futuras adversidades.

En una palabra, nos corresponde examinar el establecimiento de las bases de un sistema de cooperación económica internacional y su evolución posterior, así como la implementación de sus criterios centrales, que en definitiva se aplicaron sólo a determinados grupos de países unidos ya sea por las condiciones geográficas o inquietudes semejantes.

Tal como se dijo recientemente, el problema del comercio internacional fue el que ocupó el primer lugar de las preocupaciones; obviamente, los acuerdos sobre esta materia suponían necesariamente avanzar en otros campos que, si bien estaban ligados al comercio, tenian menos importancia hasta ese momento. Esto incluye lo relativo a las inversiones internacionales y el tratamiento de las materias primas.

EI punto es importante de considerar teniendo presente que en la investigación se considera de una manera especial la cooperación latinoamericana surgida mediante el establecimiento de los procesos de integración económica, materia de un ensayo posterior.

Volviendo al análisis de las situaciones de poder en que se fundaban las posiciones nacionales, constatamos que las condiciones para la asunción de Estados Unidos a un papel rector en esta esfera eran inmejorables. Las economías de los competidores comerciales y financieros de Estados Unidos, principaImente Gran Bretaña, Alemania y Japón, especialmente los dos últimos, estaban destruidas y solamente tenían esperanzas de recuperación pronta si podían contar con una extensa cooperación norteamericana. Mediante el Plan Marshall y otros programas -inicialmente no limitados en su alcance a los países occidentales- Estados Unidos prestó la asistencia financiera requerida y, junto con estimular su propia economía, abrió nuevas oportunidades de inversión en el extranjero a las empresas industriales, mineras y petroleras y de operaciones financieras en el exterior a la banca norteamericana. Similar efecto tuvieron los ele- 
vados gastos militares norteamericanos en Europa Occidental desde la década de 1950, que contribuyeron a superar lo que anteriormente se había considerado como una seria situación de "escasez de dólares"12.

Las condiciones del acceso norteamericano a fuentes extranjeras de matcrias primas - cada vez más necesarias para su economía, dado su rápido ritmo de expansión y el agotamiento progresivo de las reservas dentro de sus fronteras- constituyeron un problema clave para la estrategia económica de postguerra.

El capital europeo, tradicionalmente dominante en la economía internacional de las materias primas, debía verse desplazado en forma creciente por los intereses norteamericanos. Entre 1945 y 1970 , se acentuó aun más el liderazgo económico internacional de Estados Unidos, ya ejercido, si bien a título de emergencia, durante la Segunda Guerra Mundial. Frente a los demás países desarrollados, la posición rectora de Estados Unidos implicaba una sujeción a sus objetivos y políticas económicas, casi incondicional al comienzo, para más adelante transformarse en una especie de "dependencia negociada". Para los países en desarrollo, especialmente de América Latina, el liderazgo norteamericano significó su inclusión en un sistema político-económico global, en el cual su principal misión consistía en proporcionar materias primas industriales, combustibles y otros productos para satisfacer los requerimientos de las economías industrializadas, adquiriendo a cambio productos manufacturados. Si bien desde el punto de vista del gobierno norteamericano el sistema podía no parecer centralizado ni imperialista, las percepciones al nivel de los países subdesarrollados debian forzosamente ser distintas, ya que la situación común en cada país era la de la existencia de una o un pequeño

15Véase Robert Triffin, El Caos Monetario, México, D.F. Fondo de Cultura Económica, 1961, cap. 2. Eventualmente, los flujos de dólares de Estados Unidos al resto del mundo por concepto de programas de ayuda al exterior, pactos militares y remesas privadas contribuyeron a desequilibrar la balanza de pagos. Paradojalmente, sin embargo, tales flujos terminaron en cierto modo beneficiando intereses norteamericanos, al ser canalizados en parte por sucursales de bancos norteamericanos, en beneficio de empresas multinacionales, de la misma nacionalidad, que buscaban establecer o ampliar operaciones en Europa. Véase U. S. Senate, Committee on Labor And Welfare, "The Multinational Corporation and the Public Interest", Washington, D. C., U. S., Government Printing Office, 1973. Este informe, escrito por el profesor Robert Gilpin, sostiene que la inversión extranjera de Estados Unidos ha sido excesiva y ha ido en último término en detrimento del desarrollo económico interno de ese pais. Gilpin propone, entre otras medidas, eliminar los incentivos tributarios o la reinversión de utilidades en el exterior. 
número de poderosas empresas extranjeras, las que generalmente contaban con múltiples medios de influencia al interior de cada sistema nacional, así como con el apoyo de la diplomacia norteamericana (o de algunos otros países occidentales en ciertos casos). No fue casualidad que en este contexto eventualmente llegaran a surgir en América Latina doctrinas, teorías e ideologías político-económicas sobre las relaciones entre "centro" y "periferia", así como numerosos planteamientos sobre imperialismo y dependencia ${ }^{13}$.

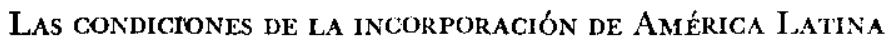

\section{EN EL SISTEMA}

Para los principales estados latinoamericanos, no se trataba, sin enbargo, de una mera "inclusión" en un nuevo orden internacional en posiciones puramente pasivas. Como señala Baldev Rilj Nayar en un ensayo de importancia fundamental, es necesario considerar la situación internacional de los países en desarrollo mediante una "unión explícita" del análisis del desarrollo con la política internacional14. De no adoptarse esta perspectiva, el comportamiento internacional de los gobiernos queda reducido a una serie de movimientos diplomátiticos que deja a oscuras la naturaleza de los intereses y objetivos en

${ }^{13}$ Desde el artículo de Osvaldo Sunkel, "Polftica Nacional de Desarrollo y Dependencia Externa" Estudios Internacionales, I No 1, 1967, han sido numerosos los análisis inspirados en la tesis de la dependencia, tanto dentro como fuera de América Latina. Véase, por ejemplo, Fernando $H$. Cardoso y Enzo Faletto, Dependencia y Desarrollo en América Latina. Buenos Aires, Siglo xxi, 1969 y Helio Jaguaribe y otros, La Dependencia Politico Económica de América Latina, México, D. F. Siglo xxi, 1969.

En un prolijo análisis estadístico de relaciones bivariadas, tomando como variables independientes diversas medidas de dependencia comercial y de capital, Robert Kaufman y otros han demostrado la complejidad del problema económicopolítico de la dependencia. En particular, Kaufman y sus colegas demuestran la implausibilidad de la tesis del estancamiento económico como consecuencia inevitable de la dependencia, lo que dejaría, sin embargo en pie muchas de las tesis del llamado "desarrollo dependiente". Los autores concluyen que el "peso de la prueba" en todo caso, debería pasar a los sostenedores de las tesis de la dependencia, junto con enfatizar la necesidad de estudiar mís detenidamente diversos tipos de dependencia, así como variables que intervienen entre la dependencia y sus efectos postulados. Véase Robert Kaufman y otros, "A Preliminary Test of the Theory of Dependency" Comparative Politics Vol. 7 No 3, Abril 1975, 303-330, especialmente pp. 318 y sigtes.

"Baldev Raj Nayar, "Political Mainsprings of Economic Planning, in the New Nations., Comparative Politics, Vol. 6, No 3, Abril 1974, p. 345. 
juego. Dentro de este enfoque, y coincidiendo parcialmente con el análisis de Nayar, es posible sostener que para los principales estados latinoamericanos desde el período de la Segunda Guerra Mundial, no habia alternativa frente al "imperativo de la moderniza. ción", el cual emergió poderosamente por una conjunción de factores, entre los cuales fue importante, en primer lugar, la percepción de amenaza a la seguridad nacional creada por la condición de atraso económico ${ }^{15}$. En el caso latinoamericano, tal percepción se dio claramente en Brasil, Argentina y México, cuyos dirigentes políticos desconfiaban de Ias potencias, que se disputaban el dominio del sistema internacional desde antes de la guerra, sin perder al mismo tiempo de vista las oportunidades de negociación que surgían al amparo de las grandes rivalidades internacionales. En el caso de Brasil, por cjemplo, el Presidente Vargas por largo tiempo mantuvo un delicado equilibrio entre tendencias contrapuestas pronorteamericanas y proalemanas en pugna en su gobierno, tratando de explotar cl potencial apoyo diplomático brasileño a uno u otro pás para comprometer a Estados Unidos o Alemania en los más importantes proyectos del desarrollo industrial del Brasil, especialmente la construcción de una gran industria siderúrgica. La relación entre subdesarrollo y amenazas a la seguridad queda claramente ilustrada en cste caso. En efecto, una de las principales consideraciones por parte del gobierno brasileño al querer emprender la creación de una industria nacional del acero, fue la voluntad de reducir la dependencia de productos de acero importados, que eran vitales para diversas actividades cconómicas de carácter estratégico. También es interesante la situación que comentamos desde el punto de vista de la relación cntre política exterior e interna, puesto que los sectores favorables al acercamiento a cada una de las potencias en pugna cran al mismo tiempo los que impulsaban proyectos globales de desarrollo nacional para Brasil que se enmarcaban dentro del "campo ideológico" representado por Estados Unidos y Alemania. Los partidarios del acercamiento a Estados Unidos favorecian una política de desarrollo de corte liberal, en la cual los inversionistas privados nacionales y extranjeros debían ser los actores principales. Los sectores proalemanes, por el contrario, favorecian una política de desarrollo de carácter marcadamente estatista, en que debía corresponder un papel primordial a las fuerzas armadas. Fueron éstas,

${ }^{15}$ Nayar, op. cit., p. 346. 
en definitiva, las que habrían de controlar la industria del acero, pues, al no prosperar un proyecto de inversión de la empresa U.S. Steel, intervino el gobierno, obteniendo financiamiento público norteamericano para la construcción del complejo de Volta Redonda, organizado como empresa pública autónoma. Desde el punto de vista norteamericano, la política de Vargas no contradecía los intereses privados, ya que la organización de esta industria básica con la asistencia de Estados Unidos aseguraba importantes corrientes futuras de intercambio, desplazando la competencia alemana. Para Brasil, las condiciones ventajosas obtenidas de Estados Unidos debían a su vez influir en las opciones diplomáticas nacionales: en 1942, el gobierno de Vargas rompió sus relaciones diplomáticas con los países dcl Ejc, y comenzó a participar activamente desde entonces en la alianza bélica. No obtuvo, sin embargo, la participación a que aspiraba en la planificación del sistema internacional de postguerra ${ }^{16}$.

Evidentemente, las consideraciones de seguridad nacional que subraya Nayar no fueron las únicas que contribuyeron a crear consenso en torno al imperativo de la industrialización. La depresión de comienzos de la década de 1930 jugó un papel importante en el mismo sentido, al producir graves disminuciones en los ingresos latinoamericanos provenientes de exportaciones de productos agrícolas y materias primas a los pafses industriales. Tales ingresos eran indispensables para el financiamiento de importaciones esenciales para la región. La "sustitución de importaciones" por productos nacionales debía ayudar a enfrentar esta seria situación, que en la década siguiente se vio nuevamente agudizada bajo las presiones económicas de la guerra.

Un tercer factor de impulso a las politicas latinoamericanas de industrialización lo constituyeron las "coaliciones populistas" que gobernaron en los principales estados latinoamericanos entre las dé. cadas de 1930 y 1950, desplazando del control exclusivo del poder a las fuerzas políticas tradicionales, cuyo poder había descansado fundamentalmente en la tenencia de la tierra, la minería, el comer-

${ }^{20} \mathrm{El}$ desarrollo de las relaciones internacionales brasileñas durante la Segunda Guerra Mundial es estudiado en detalle en la obra de Frank Mc Cann, The Brazilian-American Alliance, 1937-1973, Princeton, N. J., Princeton University Press, 1973, John D. Wirth en The Politics of Brazilian Development, 1930-1954, Stanford, Stanford University Press, 1970, presenta un interesante análisis de Ia política de desarrollo de la industria del acero bajo el gobierno de Getulio Vargas. 
cio internacional y las finanzas. El advenimiento de regímenes "populistas" cuyas características responden al llamado "pretorianismo radical" de Samuel Huntington ${ }^{17}$, estuvo asociado con el proceso de urbanización y la creciente complejidad de las estructuras sociales de los pafses latinoamericanos.

Según ha demostrado Guillermo O'Donnell, las "coaliciones populistas" que resultaron de la incorporación de nuevos sectores sociales a la actividad política, buscaron expandir sus aún precarias bases de poder en los principales estados sudamericanos, recurriendo al estado como promotor de sus intereses ${ }^{18}$. L as clases medias en cstos paises no se caracterizaban jor su dinamismo empresarial, y representaban más bien sectores sociales en ascenso, interesados en la protección gubernamental para sus "conquistas", que las diferenciaban tanto de los sectores tradicionales como de los estratos subordinados $^{19}$. I os intereses de estos sectores requerian un abandono progresivo de las políticas económicas exteriores de orientación librecambista, favorecidas por los regímenes tradicionales, y la erección de barreras proteccionistas que permitieran la formación de mercados internos suficientemente grandes y estables para absorber la producción de las industrias nacionales, fundamentalmente de bienes de consumo, así como de algunos biencs durables. No obstante, no se trató de una opción tajante entre dos políticas opuestas ya que, al mantenerse los requerimientos de importación que en esta fase incluirian proporciones cada vez más altas de materias primas y bienes de capital para la industria, era indispensable no perjudicar las perspectivas del sector exportador. Debía quedar espacio, por consiguiente, para efectuar compromisos políticos dentro de la orientación general descrita ${ }^{20}$.

Desde luego, la política de industrialización exigió, más allá de lo ya señalado, el cstablecimiento de un sector público oricntado a

rVéase Samuel P. Huntington, Political Order in Changing Societies, New Haven, Yale University Pıess, 1968, cap. 4, para un antílisis de la polf́tica "oligár. quica", "radical" o "de masas" como fases del desarrollo --y de la decadenciade los regímenes politicos en los países en desarrollo.

${ }^{18}$ Guillermo O'Donnell, Modernizarión y Autoritarismo, Buenos Aircs, Paidós 1972, cap. 2.

${ }^{29}$ Uno de los mejores andisis de este punto es el de Lais Ratinoff, "The New Urban Groups: "The Middle Classes" en Seymour M. Lipset y Aldo Solari, eds. Elites in Latin America, Nucra York, Oxford University Press, 1967, cap. 2.

s'O'Domell, op. cit., p. 56. 
proveer la infraestructura física así como algunos insunos básicos para el sector industrial. A cllo respondieron las políticas de desarrollo de las industrias siderúrgicas, cono en el caso comentado más arriba, las políticas de electrificación y otros aspectos de las políticas energéticas, etc. Todas estas tarcas no cabian dentro del horizonte de los sectores privatos nacionales, dadas sus caracteristicas tradicionales y su bajo gado de dinamismo. Ias corporaciones públicas de desarrollo que surgicron en diversos países de la región desde que el gobicrno del Presidente Pedro Aguirre Cercla creara la Corporación de Fomento de la Producción en Chile, fucron el instrumento preferido de los gobiernos para promover la creación, tanto del sector público de industrias básicas como el apoyo a la empresa privada. Al mismo ticmpo, estas instiluciones introdujeron los primeros intentos de planificación ecomómica y realizaron importantes esfueros para desirrollar fuentes de información sobre problemas económicos en sus respectivos países.

I.o anterior no debe entenderse, sin embargo, en el sentido de que se hayan diseñado e implementado estrategias coherentes de desarrollo nacional, centradas en la industrialización para sustituir las importaciones de manufacturas. I as politicas seguidas fueron parciales, las inversiones insuficientes, y los objetivos estuvieron centrados sobre todo en la sustitución "fácil" de importaciones en mercados que en todo caso eran demasiado reducidos para la realización de economias de escala ${ }^{21}$. Ia inestabilidad de los ingresos provenientes de las exportaciones no permitia acometer en condiciones adecuadas el financiamiento nacional de proyectos más ambiciosos y, por otra parte, la creación de sectores trabajadores ubanos politicamente movilizados incentivó desde un comienzo a los gobicrnos interesados en su apoyo, a enfatizar los proycctos que pudieran traducirse a corto plazo en la satisfacción de nuevas expectativas de empleo y consumo orientadas por los modelos provenientes de los países occidentales más desarrollados. La combinación de las crisis de recursos extcrnos con estas demandas por mejoramientos inmediatos eventualmente habria de traducirse en crisis conómicas y problemas de incstabilidad politica y en refinitiva, en la década

stAlberto Picdra, "Problems of Fortigu Ecomomic Relations" cu Davis y Wilson, eds., op. cit., p. 39 , cita en cete sentido al profesor Stefan Robeck Indus. trialization through Import substituion of Expont Industries: A False lichotomy, en J. W. Markham y G. Papanck, cels. Industrial Organization and Fronomic Development, Bostos, Houghton Miffiu Cu., 1970, pp. 354-355. 
pasada, como señala O'Domell, en el agotamiento de las opciones "populistas" con sus estrategias de desarrollo"2.

Las dimensiones externas de la participación Iatinoamericana en el sistema de postguerra han sido tratadas en parte anteriormente. A lo dicho allí cabe agregar solamente dos puntos que son, sin $\mathrm{cm}$ bargo, cruciales para comprender correctamente la nueva posición latinoamericana en el sistema internacional desde 1945 .

EI primero se refiere a lo que podríamos calificar como una nueva forma de relación política internacional, centrada en los problemas económicos.

Ia estructuración de los compromisos que establecieron las nuevas bases de una cooperación internacional requerian, necesariamente, una readecuación tanto en la substancia de los compromisos como en la manera jurídico-institucional de hacerlos cfectivos. Desde este ángulo, se sientan las bases para un derccho económico internacional, que se diferencia del derecho internacional clásico en la medida que fuerza en el instante mismo, a compromisos especificos. Las derivaciones jurídicas y politicas de este hecho han sido múltiples. La principal, quizás, ha sido la base teórica y práctica para el nacimiento del derccho de integración cconómica, que ha

20'Dounell, op. cit., pp. 60 y siguientes. Por e'slar thís allí del tema de este trabajo, no entramos en cl texto on la discusión de las alternativas que sobrevienen en la situación descrita. Hásicamente, puerte decitse que en Brasil cspecialmente y en Argentina, el agotamiento de las fórmulas populistas favorece el surgimiento de una pugna entre lo que Huntington llama el "pretorianismo de masas" y las fuerzas que llevan al pexler a regímenes militares "institucionales" de características "lurocrático-autoritarias" (O'Domell). Fı Brasil se dio un claro movimiento hacia este tipo de régimen, nicntras on $A$ rgentina se ha dado una "alternación" o rotativa. El caso chileno hasta ahorit no ha sido sistemáticamente analizado en estos términos. Cale siaturalmente pregruntarse si la opción "burocrático-autoritaria" representia una tendencia estable, o si es meramente una más dentro de las muchas fótmulits que, c'u definitiva, son desplazadas. Sobre este debate, véase Guillermo O'Donnell, "Corporatism and the Question of the State", on James Malloy, ed.. Aluhoritarianism and Corporatism in Iatin America, Pittsburgh, University of Pittsburglı Press, 1977, cap. 3, y Douglas A. Chalmers, "The Politicized State in Iatiu America", en Malloy ed., of, cit., cap. 2. El sociólogo Alejandro P'ortes, contraponicudo los conceptos de "modernidad" y "desarrollo", ha sostenido an un importante artículo que una raí del subdesarrollo latinoamericano se encuentra en el desfase curre aspiraciones subjetivas adquiridas por la vía de la socíalización, y lat ralidad sociopolítica que impide la satisfacción de las demandas. Yrer $A$ lejiudere Portes, "Modernity and Development: A Critique", Studies in Comparative Intonational Development vir $\mathrm{N} \odot 3$, Otoño 1973, 217-279. 
conocido un desarrollo de enorme importancia en las diversas experiencias regionales y que, a pesar de sus limitaciones ha traído una nueva era de política internacional "intralatinoamericana", comenzando también a ponerse en cuestión la concepción clásica de la soberania estatal desde que un conjunto de paíscs deciden libremente formar parte de agrupaciones destinadas a favorecer el desarrollo de los intereses nacionales.

Ia forma de la cooperación internacional que se estableció, exigia conductas fielmente seguidas por los países. Especificamente, la carta de La Habana no es más que un código de conducta de carácter amplio desde el punto de vista temático. Es notable ver cómo en la actualidad han sido los países de América Latina los líderes en el planteamiento de la necesidad de establecer códigos de conducta internacionales relativas a determinadas materias. A estc respecto, las experiencias desarrolladas con éxito (al menos en su dimensión jurídico-económica) como es el caso de las normas sobre inversiones extranjeras y transferencia de tecnología en el Grupo Andino, han estimulado la extrapolación de estas experiencias al campo de los trabajos de unCTAD. Hay, por lo tanto, un interesante fenómeno de interacción entre los niveles global y regional en la formulación e implementación de posiciones y demandas relativas al orden económico internacional. Nuestra hipótesis de la adhesión formal de América Latina al orden internacional de postguerra debe ir acompañada, por tanto, de una exploración de las fucntes de la innovación en este orden de cosas, que también suele surgir en la región e incluso ha llegado a producir movimientos favorables a determinados cambios en el nivel global.

Es de destacar, sin embargo, la línea esencialmente tcórica de muchas concepciones y su alejamiento de las políticas nacionales seguidas en los principales países de América Latina, hasta que en algunos centros se imponen criterios más prácticos para la formulación de posiciones.

La dispersión de problemas hace que, si bien por un lado el planteamiento de soluciones de esta naturaleza en la actualidad aparezca como de gran valor en sí mismo, su implementación requiere la inserción en un marco homogéneo de políticas cconómicas. Ello, junto con dificultar las soluciones prácticas de aplicación, exige, en forma previa, un compromiso sobre materias de gran sensibilidad.

La Carta de La Habana presentó la especial particularidad de combinar códigos de conducta de gran exigencia y compromiso, 
dejando al mismo tiempo la implementación de las soluciones entregada en gran medida al cumplimiento nacional. Como se verá más adelante, la confrontación entre los temas de "estricto compromiso" y los de "amplio compromiso" permite ciertas deducciones de gran interés y que permiten, al mismo tiempo, comprobar que la "sensibilidad" de algunas materias se ha mantenido en estos treinta últimos años.

Esa confrontación temática puede, además, tener el mérito de señalar determinadas materias cuya solución debe, necesariamente, establecerse si se quiere llegar en forma expedita y seria al establecimiento de un Nucvo Orclen Económico Internacional justo y política y juridicamente operable.

Nuestra discusión de las condiciones políticas quedarfa incompleta, sin embargo, si no entráramos en un breve análisis de lo que podría llamarse la progresiva "marginalización" económico-política en América Latina entre el fin de la guerra y comienzos de la década de 1960 .

En síntesis, el proceso de "marginalización" consistió, en el nivel político, en la relegación de la región a un plano secundario en las prioridades políticas norteamericanas; en el nivel económico, en la disminución de la proporción del comercio internacional de los países de la región dentro del intercambio general, y en la pérdida de importancia relativa de América Latina como mercado para las inversiones extranjeras, especialmente las provenientes de Estados Unidos. Pero tal "marginalización" no significó una atenuación de la dependencia económica, puesto que a pesar de la baja relativa en la importancia económica de la región para los países industrializados, éstos, sobre todo Estados Unidos, expandieron su rol económico, manteniendo una participación decisiva en las principales decisiones económicas que afectaban a los países de la región tanto desde fuera como desde el interior de cada uno de los sistemas po. líticos ${ }^{23}$.

Hans J. Morgenthau, refiriéndose a la oposición norteamericana a la política de "esferas de influencia", señala que las reservas de Estados Unidos a esta forma de dominación internacional, desaparecen cuando ésta sirve manifiestamente los intereses nacionales de

23" Una nación dependiente (en tírminos económicos) es aquella que carece de control sobre las principales decisiones que afectan su propia economia" Barbara Stallings, "Economic Dependency in Africa and Latin America", Sage Professional Papers, Comparative Politics, Series 01-031-1972, p. 6. 
ese país. Concretamente, "la Doctrina Monroe que, estipula la exclusión de las instituciones politicas europeas y las adquisiciones territoriales del hemisferio occidental, y de esta manera deja libre juego a la preponderancia de Estados Unidos, es la proclamación más amplia y unilateral de una esfera de influencia de los tiempos modernos". Los estadistas norteamericanos no han vacilado en referirse al hemisferio occidental o a parte de él, como una csfera de influencia americana"2t. Morgenthau y Herbert Feis concluyen que al respecto no cabía discusión dentro de la alianza occidental, pues no fue otro que Churchill, quien, durante la guerra ratificó que en Sudamérica los ingleses "seguimos lo más posible la conducción de Estados Unidos, mientras no se trate de nuestro vacuno y nuestro cordero. Sobre esto naturalmente tenemos puntos de vista muy definidos, debido a lo poco que recibimos" 25 .

El interćs fundamental de Estados Unidos, dentro de la baja prioridad asignadia a los asuntos latinoamericanos, era la mantención del statu quo. Se trataba no tanto de una diplomacia "imperialista", sino "imperial" aunque el grado de dominación alcanzado por Washington nunca fue completo, sobre todo en relación con los cstados principales, que conservaron cierta capacidad de maniobra.

Para los gobiernos de la época, Ia mayor preocupación era detener el "pcligro comunista" y asumir la conducción de la defensa hemisférica, "ayudando a ciertos estados latinoamericanos, dentro de los más grandes, a hacer una contribución mediante la provisión le ayuda militar" 20 .

En realidad, la colaboración latinoamericana excedió el ámbito hemisférico. Estados Unidos pudo durante largos años contar con el "bloque latinoamericano" en las votaciones de la Asamblea General de las Naciones Unidas, mientras ese bloque fue el grupo regional más fuerte, controlando más de un tercio de la votación total. Uno de los primeros análisis de las votaciones en la Asamblea General reveló que "de las 21 repúblicas americanas que comprende la of.A,

${ }^{2}$ Hans J. Mongenthau, ensayo en Lloyd Gardner, Arthur Schlesinger Jr. $y$ Hans J, Morgentliau, cusayo on Lloyd Gardncr, Arthur Schlcsinger Jr. y Hans J. Morgenthat, Origins of the Cold War Waltham, Mass., Ginn. Blaisdell, 1970, pp. 86-87.

${ }^{2}$ Sir Winston Churchill, Triumph and Tragedy, pp. 74-75, citado por Morgenthau, op. cit., p. 87 y Feis, op. cit., p. 341.

${ }^{n g}$ Larman C. Wilson, "Multilateral Policy and the oss", en Harold E. Davis y Larman C. Wilson, eds. Lalin American Foreign Policies and Analysis, Baltimore, The Johns Hopkins Lniversity Press, 1976, p. 53. 
dos tercios o más de los estados participantes en cada votación estaban en el mismo campo... en los asuntos sustantivos; el peso de los votos latinoamericanos es mís importante que cualquier combinación de bloques teóricamente posible"2-2. Naturalmente, los estados Iitinoanericanos que cumplían períodos en el Consejo de Seguridad también volitban junto a Estados Unidos en forma regular: como scñalaba Dean Acheson en sus memorias, "Los latinoamericanos habían sido particularmente leales en las crisis de Corca, Cuba y Ecuador; emiticron rotos cruciales para las resoluciones del Consejo de Seguriclad los chías 25 y 27 de junio de 1950, y el Consejo de la oEA el 28 de junio de 1950 apoyó las decisiones de las Naciones Unidas, y comprometió la solidaridad continental. En febrero de 195I, las repúblicas americanas apoyarian la resolucion condenando a los chinos commistas, y poco después de la Cuarta Reunión de Consulta, apoyarian tambión el embargo de matteriales estratégicos"28.

En los asuntos hemisféricos, el apoyo latinoamericano a las causas políticas de Estados Unidos fue movilizado en diversas oportunidades, siendo desde lucgo la mís notable, la declaración de incom. patibiliclad de la existencia de regímenes comunistas en la región, con la mantención de la paz en el sistema interamericano, hecha en la Conferencia de Caracas en 1954, ante el problema de Guatemala. Fue necesario que surgierat el espectro revolucionario y antinorteanericano en la región, para que pudiera ocurrir un cambio profundo en el enfoque político de Washington, cambio que involucraba un viraje frente a las viejas denandas regionales de reformas a las relaciones cronómicas. Pero itun dentro de esta nueva posición -que llevó a la Alianza para el Progreso, tras un período de reevaluación en el áltimo año de gobierno de EisenhowerWashington se vio simultíneamente haciendo esfuerzos hacia el desarrollo y cl progreso y para impedir la subversión y la insurgencia ${ }^{29}$.

Este duilismo de lit política norteamericana naturalmente debía introducir un elemento de inestabiliclad en las relaciones hemisfé. ricas.

"iM. Margatet Ball, "Bloc Voting in the General Assambly", International Organizacion Vol. 5 V 1 1. 1951, reproducielo en Lcland Goodrich y David Kay, eds. Intemational Organization: Politics and Process, Madison, University of Wisconsin Press, 1973, pp. 102 y 101.

sDean Aclieson. Present at the Creation My Yeats in the State Department, Sueva Yorh. Sew American librats, 1969, pp. 610-641.

"Stanley Ioffmam, Gulliv's Troubles, or the Sctting of American Foreign Policy, Nueva Yotk, II G Gaw Hill Book, Co., 1968, p. 187. 
Pero una somera revisión de las relaciones económicas regionales muestra, sin perjuicio de la mayor relevancia política que tuvo América Latina en la década pasada, una progresiva "marginalización" económica en el sistema internacional. Este proceso se explica por el extraordinario crecimiento del comercio entre los países altamente industrializados, junto con el menor dinamismo de América Latina en el comercio internacional en comparación con otras regiones en desarrollo. Mientras entre 1960 y 1970 las exportaciones de todos los países en desarrollo aumentaron en una tasa media anual de $7,4 \%$, las de los paises latinoamericanos aumentaron solamente en un $5,0 \%$ anual. Como consecuencia de este menor ritmo de expansión, la proporción de las importaciones de los países industrializados originarias de los países en desarrollo bajó de $24 \%$ en 1960 a $18 \%$ en 1970, y la proporción proveniente de América Latina de $8 \%$ a $5 \%^{30}$. En 1950, en cambio, la proporción de las exportaciones mundiales por los países en desarrolto habia sido de $32 \%$, lo que demucstra la existencia de una disminución importante de su participación en los mercados internacionales en los años siguientes ${ }^{31}$.

En el mercado nortcamericano, este proceso, que fue allí particularmente agudo, hizo bajar la participación latinoamericana en las importaciones de $24 \%$ a sólo un $11 \%$ en la década pasada. La caída de la participación del Tercer Mundo en dichas importaciones en el misno lapso fue de un $40 \%$ a un $25 \%$-en otras palabras, se explica la caída casi exclusivamente como consecuencia del estancamiento latinoamericano ${ }^{32}$, el cual, a su vez, se produjo como consecuencia de políticas comerciales regionales y de un cambio en la composición de las importaciones de Estados Unidos (consistente en un aumento relativo de las importaciones de manufacturas) ${ }^{33}$.

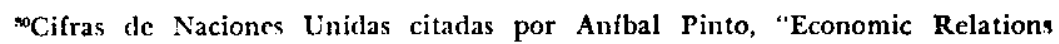
Between Latin America and the United States: some Implications and Perspective", en Julio Cotler y Richard Fagen, eds. Iatin America and the United States The Changing Political Realities, Stanford, Stanford University Press, 1974, p. 101, Tabla 1.

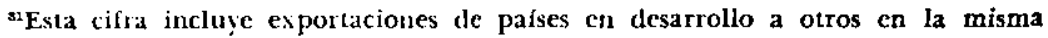
categoría, pero éstas ctan muy bajas en 1950, y aún a fines de la década pasada, apenas alcanzalsan 3-4\% del comercio mundial, según el Informe Pcarson, Partners in Development Report of the Commision on International Development, Nueva York, Preager Publishers, 1969, p. 91. La cifra de 32\% para 1950 se basa en un informe del Banco Mundial citado por Piedra, op. cit., p. 26.

apinto, loc. cit.

sobviamente, las tendencias señaladas son muy globales. Pinto señala la exis- 
Al considerar además el rol de las inversiones extranjeras en la región se advierte, como indica Aníbal Pinto, "la discontinuidad entre el crecimiento de las obligaciones financieras de América Latina y la marginalización relativa de la región del mercado de Estados Unidos" 34 . En efecto, durante el periodo de postguerra se advierte, junto al relativo debilitamiento de la interdependencia comcrcial, una creciente vinculación establecida por la vía de las inversiones extranjeras, especialmente inversiones directas de origen norteamericano. Mientras las exportaciones permanecen más o menos estacionarias, los pagos a Estados Unidos por concepto de remesas de utilidades de inversiones directas y dividendos de carteras de inversión suben considerablemente, no obstante que las inversiones norteamericanas en la región crecen menos que las que se efectúan en otros países industrializados por empresas de Estados Unidos, especialmente en Europa Occidental y Canadá.

Theodore Moran, entre otros autores, ha presentado datos relativos a la industria manufacturera que tienden a apoyar esta aseveración. En 1950, las inversiones norteamericanas en el sector manufacturero latinoamericano totalizaban us\$ 781 millones, lo que representaba un $20 \%$ del total de las inversiones extranjeras de Estados Unidos en dicho sector de la actividad económica. En 1957, a pesar que la inversión subió a us\$ 1.280 millones, ella solamente representaba un $16 \%$ de las inversiones norteamericanas en manufacturas en el mundo. En 1969 el valor de las inversiones regionales llegó a us $\$ 4.347$, pero se trataba solamente del $15 \%$ del total invertido en el mundo, puesto que esta última cifra bordeaba los us\$ 30.000 millones $^{35}$. En otras palabras, la importancia relativa de América Latina como área receptora de inversiones norteamericanas tiende a bajar, pero desde la perspectiva latinoamericana la depen. dencia respecto a las inversiones extranjeras aumenta.

\section{Conclusiones PRELIMinares}

El análisis precedente ha dejado de manifiesto, a través del estudio de algunos aspectos del sistema internacional, la magnitud de los

tencia de algunas tendencias que deberían ser tomadas en cuenta en análisis cconómicos detallados, especialmente distinguiendo grupos de palses y tipos de exportaciones.

suPinto, op. cit., p. 109.

Theodore Moran, "Commentary on Martins", en Cotler y Fagen, eds. op. cit., p. 405. 
caminos en las condicionantes de la participación política latinoamericana en los asuntos mundiales. En general, dichos cambios apuntan hacia una disminución de las opciones internacionales de los palses de la región como consecuencia de la Segunda Guerra Mundial y la Guerra Fría. La relación entre el posterior mejoramiento de las relaciones entre las grandes potencias y el comportamiento internacional de América Latina no ha sido tratada en este estudio.

En general, las politicas latinoamericanas en el perlodo en estudio se orientan en torno a establecer una interrelación entre politicas exteriores y objetivos internos, de modo que aquéllas sirvan a los fines de modernización nacional y en especial de industrialización que persiguen los gobiernos de la región, bajo diferentes fór. mulas politicas. Sin embargo, el surgimiento de nuevos estados y la recuperación económica-política de los pafses occidentales aliados de Estados Unidos afecta negativamente el peso de la región dentro del bloque occidental ${ }^{96}$, en el cual América Latina ocupa en el perfodo en estudio, un lugar relativamente secundario, al no tener una alta prioridad estratégica ni económica para las grandes potencias. Esta situación cambia solamente al advertírse en America Latina y Estados Unidos que existen fuerzas internas e internacionales favorables a un cambio del statu quo, y que podran llegar a poner en tela de juicio algunos aspectos del rol hegemónico norteamericano.

En este periodo, América Latina asiste, sin ser más que un interesado espectador, al establecimiento de un orden económico in. ternacional diseñado por las grandes potencias y que responde al consenso que entre éstas surge en torno a una filosofía económica internacional.

Tan modesto rol explica en parte el hecho de que la adhesion latinoamericana a los principales acuerdos económicos haya sido principalmente formal. No obstante, durante el perfodo de postguerra, en la medida que la implementación de los mecanismos entra en alguna medida en conflicto con las aspiraciones y requerimientos de algunos estados de la región, comienzan a manifestarse posiciones

*Un ejemplo concreto paede verse en los antecedentes de la formación del Merado Común Centroamericano, especificamente en lo referido a determinados sectores de producción (textil) que se desarrollaron extraordinariarnente durante la Segunda Guerra Mundial, pero luego que se inicio la recuperación de las economfas de algunos patses orientales, comenzo el decrecimiento. Ver Wionczek (M.) Editor, Integracidn de América Latina, Fondo de Cultura Económìca, 1964. 
generales y demandas especificas tendientes a introducir modificaciones en las organizaciones económicas.

Todo esto configura un cambio en la naturaleza de las relaciones internacionales de América Latina, que pasan a asumir un carácter marcadamente económico en su contenido y progresivamente pragmático en su estilo.

Algunas de las acciones latinoamericanas al inicio del período de postguerra prefiguran, como en el caso de la insistencia en "códigos de conducta" en la Conferencia de La Habana, temas y soluciones que posteriormente reaparecen en la discusión sobre el "Nuevo Orden Económico Internacional". 